\title{
Review of the Current Management of Pressure Ulcers
}

\author{
Tatiana V. Boyko, ${ }^{1,2}$ Michael T. Longaker, ${ }^{1,3}$ and George P. Yang ${ }^{1,4,5, *}$ \\ ${ }^{1}$ Hagey Laboratory for Pediatric Regenerative Medicine, Department of Surgery, Plastic and Reconstructive Surgery \\ Division, ${ }^{3}$ Institute of Stem Cell Biology and Regenerative Medicine, and ${ }^{4}$ Department of Surgery, Stanford University \\ School of Medicine, Stanford, California. \\ ${ }^{2}$ Department of Surgery, University at Buffalo SUNY, Buffalo, New York. \\ ${ }^{5}$ Veterans Affairs Palo Alto Health Care System, Palo Alto, California.
}

Significance: The incidence of pressure ulcers is increasing due to our aging population and the increase in the elderly living with disability. Learning how to manage pressure ulcers appropriately is increasingly important for all professionals in wound care.

Recent Advances: Many new dressings and treatment modalities have been developed over the recent years and the goal of this review is to highlight their benefits and drawbacks to help providers choose their tools appropriately.

Critical Issues: Despite an increased number of therapies available on the market, none has demonstrated any clear benefit over the others and pressure ulcer treatment remains frustrating and time-consuming.

Future Directions: Additional research is needed to develop products more effective in prevention and treatment of pressure ulcers.

Keywords: pressure ulcer, pressure injury, wound dressings

\section{SCOPE AND SIGNIFICANCE}

THE FASTEST GROWING segment of our population is those over 65 years of age, and there are increased rates of obesity, diabetes, and cardiovascular disease. ${ }^{1}$ This combination of factors has resulted in more people needing assistance with activities of daily living due to decreased mobility. ${ }^{2} \mathrm{~A}$ major morbidity of decreased mobility is development of a pressure ulcer. The treatment for pressure ulcers is lengthy and causes a significant financial burden on the healthcare system. In the United States, an estimated $\$ 11$ billion dollars is spent on pressure ulcers yearly, with $\$ 500$ to $\$ 70,000$ being spent on a single wound. ${ }^{3}$

\section{TRANSLATIONAL RELEVANCE}

Despite a number of new dressings and treatments available for the management of pressure ulcers, none has been demonstrated to have a significant benefit over the other. The basic principles of maintaining the wound clean and well perfused remain the hallmarks of therapy. A major target for new therapies would be finding approaches to decrease incidence among susceptible patients, especially given potential penalties in reimbursement for patients who do develop a pressure ulcer.

\section{CLINICAL RELEVANCE}

The prevention and treatment of pressure ulcers are highly relevant to wound care professionals. These patients require prolonged course of treatment to fully heal their wounds. The biomedical burden is tremendous as noted above and healthcare expenditures on this problem are

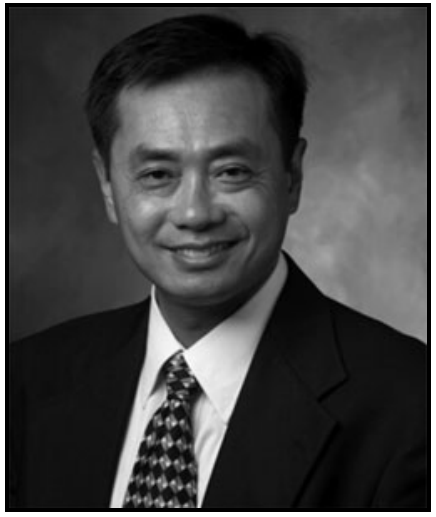

George P. Yang, MD, PhD

Submitted for publication June 7, 2016. Accepted in revised form August 5, 2016.

*Correspondence: Department of Surgery, Stanford University School of Medicine 257 Campus Drive, Stanford, CA 94305 (e-mail: gpyang@stanford.edu). 
only rising. Penalties now imposed for hospitalacquired pressure ulcers mean we need greater knowledge about causation and prevention.

\section{CAUSATION}

Pressure ulcers develop as a result of a combination of physiologic events and external conditions. The classic thinking of tissue ischemia induced by prolonged external pressure on tissue being the sole causative factor of pressure ulcer formation has been examined more systematically. Along with localized ischemia and reperfusion injury to tissues, impaired lymphatic drainage has been shown to contribute to injury as well. Compression prevents lymph fluid drainage, which causes increased interstitial fluid and waste build up and contributes to pressure ulcer development. Deformation of tissues has been shown to be a greater indicator of pressure ulcer formation than pressure exerted on tissues alone. ${ }^{4}$ The time required to develop a pressure ulcer is dependent on many factors, including the patient's physiology and the degree of pressure and shear force placed on the tissue. ${ }^{5}$ Pressure ulcers occur over predictable pressure points where bony protuberances are more likely to compress tissues when the patient is in prolonged contact with hard surfaces (Fig. 1). ${ }^{6,7}$ For patients unable to move themselves, such as intubated patients in the ICU, positional change every 2 hours has been widely accepted as effective prevention. ${ }^{8,9}$ Surgeries longer than 4 hours on a standard OR table have been shown to increase the risk of pressure ulcer formation leading to the routine use of gel pads in areas of risk during prolonged surgery. ${ }^{10}$

Pressure ulcer formation is highly influenced by risk factors (Table 1), including all conditions leading to immobility, decreased or lack of sensation, as well as malnutrition. ${ }^{11}$ Extrinsic risk factors include being immobilized on a spinal board, OR table, or bed for prolonged periods of time, as well as poorly fitted medical devices in contact with patient tissues. Intrinsic risk factors such as diabetes, malnutrition, and smoking also increase the overall risk for pressure ulcers. The spinal cord injury patient population is at the highest risk (25-66\%) of developing a pressure ulcer due to the combination of immobility and decreased sensation. A prospective study of spinal cord patients not only found that sacral and ischial pressure ulcers were very common (43\% and $15 \%$, respectively), as might be expected, but also noted that the second most common location was on the heel $(19 \%) .{ }^{12,13}$ Nursing home patients have a pressure ulcer prevalence of $11 \%$ and are most likely to develop pressure ulcers over the sacrum or heels. ${ }^{14,15}$

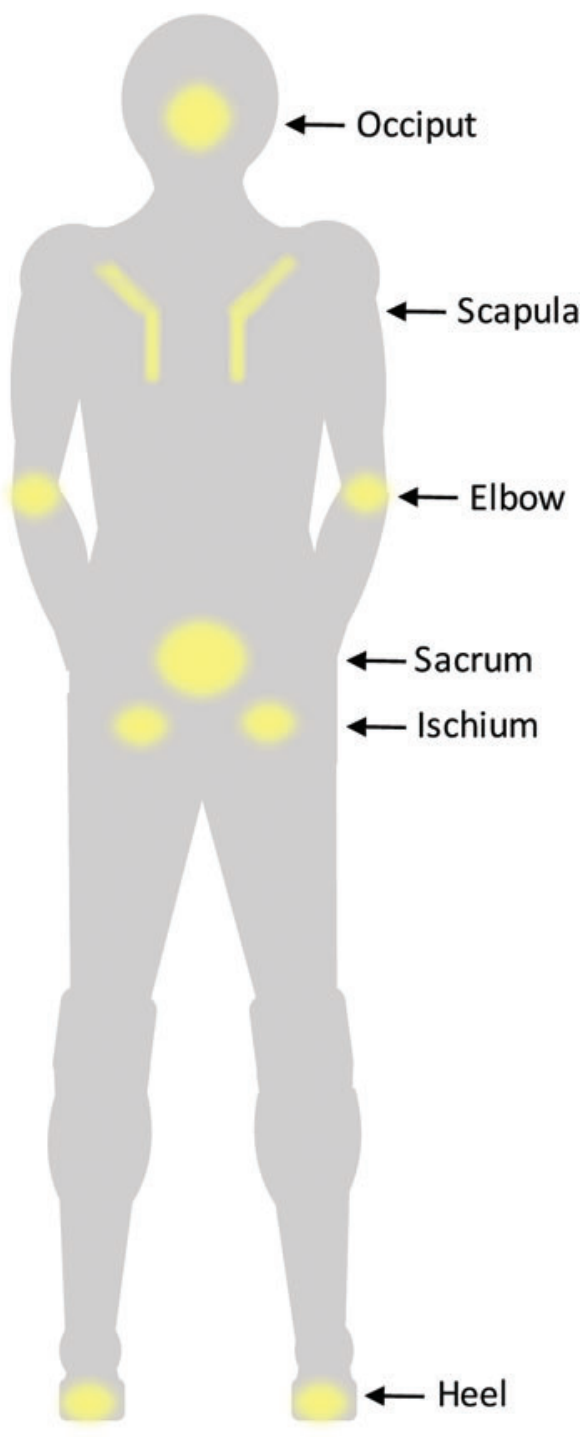

Figure 1. Illustration of locations of pressure ulcers in supine patients. To see this illustration in color, the reader is referred to the web version of this article at www.liebertpub.com/wound

Nursing home patients were also found to have contractures at a prevalence of $55 \% .{ }^{16}$ Contractures are caused by decreased elasticity of the tissue surrounding major joints, and the resulting lack of full mobility in the affected extremities significantly increases the risk of pressure ulcer formation. These data highlight how critical the need is to understand the physical, environmental, and medical risks for development of pressure ulcers in the individual patient to prevent them.

\section{RISK ASSESSMENT}

Assessing the risk for the development of pressure ulcers needs to be performed in all patients to institute appropriate prevention measures in those 
Table 1. Intrinsic and extrinsic factors influencing

the development of pressure ulcers

\begin{tabular}{l}
\hline Intrinsic risk factors for development of pressure ulcers \\
Diabetes \\
Smoking \\
Malnutrition \\
Immunosuppression \\
Vascular disease \\
Spinal cord injury \\
Contractures \\
Prolonged immobility \\
Extrinsic risk factors for development of pressure ulcers \\
Lying on hard surfaces \\
Nursing homes \\
Poorly fitting prostheses \\
Poor skin hygiene \\
Patient restraints \\
\hline
\end{tabular}

at risk. Risk should be assessed in all admitted patients as well as with any changes in mobility or medical conditions. Several risk assessment scales exist. The Norton scale scores the following five categories from a low of 1 to a high of 4: physical condition, mental condition, activity, mobility, and incontinence. A total score less than 14 indicates high risk for pressure ulcer development. ${ }^{17}$ The Braden scale is similar giving up to four points in the categories of sensory perception, moisture, activity, mobility, nutrition, and friction with a higher score indicating lower risk. ${ }^{18}$ The exact specific scale used is not as important as simply having regular risk assessment and exercising clinical judgment. ${ }^{19}$

\section{PREVENTION}

Prevention of pressure ulcer formation is directed at alleviating the risk factors for the individual patient, and is primarily focused on minimizing episodes of prolonged pressure either by placing appropriate padding at pressure points or by frequent patient repositioning. All patients using prosthetics or requiring a wheelchair for mobility should be appropriately fitted to ensure that the fit is correct and there is adequate padding. The fitting process should be repeated if there are any significant changes in weight or body habitus that can affect fit. Sweat, urine, and stool can lead to maceration of the skin and the initial skin breakdown can lead to a pressure ulcer if the skin is overlying a pressure point. ${ }^{20} \mathrm{~A}$ significant focus for care of at-risk patients is keeping the skin clean and dry. Even with adequate padding, it is important to make routine positional changes as even relatively low pressures can cause a pressure ulcer with prolonged exposure. ${ }^{5,21}$

Pressure mapping technologies have been developed to measure the amount of pressure placed on different parts of the sitting or reclining body.
These technologies have been used to develop pressure-relieving wheelchair cushions and to study normal weight-shifting behavior. ${ }^{22}$ These technologies were also used in determining that 30 degrees of wheelchair tilt is needed to relieve pressure from the ischial and sacral areas. ${ }^{23}$ Their use in bed-bound patients has been limited but has great potential for determining pressure points at risk for ulceration and in determining the effect of pressure-relieving positions on established wounds.

Any patient who has been determined to be at risk for development of a pressure ulcer or who already has a pressure ulcer needs to have a plan for repositioning. The plan needs to be individually tailored for each patient to address his or her specific needs. Frequency of repositioning needs to take many factors into account, including the support surface for the patient, general medical condition, and goals of care. Clinicians need to be cognizant of the fact that repositioning itself can create shear forces on skin, and so, the fragility and condition of the patient's skin need to be part of the assessment for how frequently to reposition them. There are a variety of factors to take into account for how to reposition patients depending on whether the patient is supine, prone, or in a wheelchair. Of special note for patients in the acute hospital setting are medical devices. Care must be taken since inadvertent positioning of the device between the patient and the support surface can create a high-pressure zone. While repositioning, lifting instead of dragging patients reduces friction and shear forces on the skin and prevents skin damage. ${ }^{5}$ Documentation of repositioning and regular skin condition assessment is key in determining early signs of pressure ulcer formation such as nonblanching erythema. System solutions such as electronic medical record programs, which prompt providers to document results of pressure ulcer screening every shift or day, are of great importance in diagnosing pressure ulcers early and preventing progression. ${ }^{24}$

A variety of pads are available, which are designed to specifically cover pressure points such as the sacrum and heels as well as foam pads designed to wrap around body parts at risk (especially feet). ${ }^{25}$ However, it is important to note that some pads can actually be detrimental. For example, supports with cutouts can have increased pressure at their edges. There are an equal number of mattress pads (egg crate mattresses, natural sheepskins, etc.) that serve to decrease pressure across a large surface area. ${ }^{21}$ Silk-based fabrics have been shown to be superior in pressure ulcer prevention when compared to 


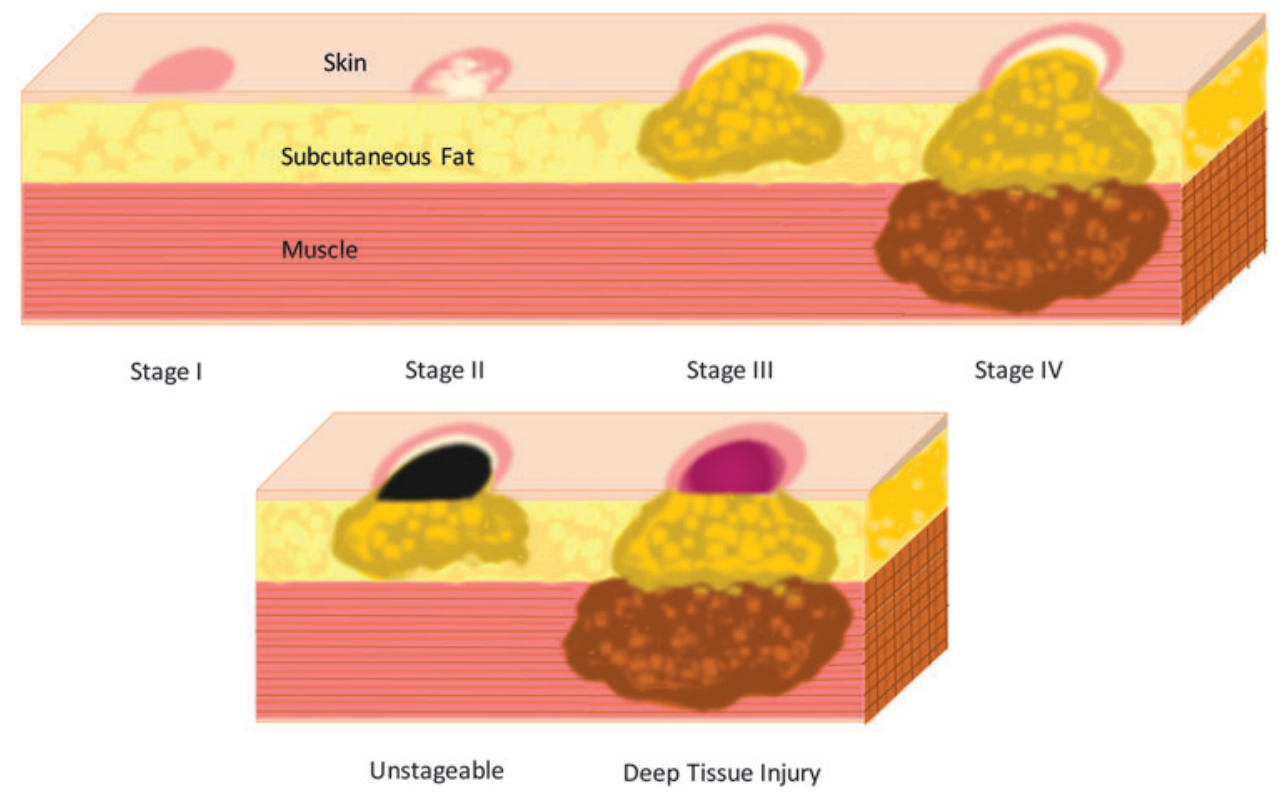

Figure 2. Illustration of different stages of pressure ulcers. From left to right. Top diagram showing pressure ulcers Stage I: skin intact. Stage II: partial skin loss. Stage III: full-thickness skin loss, subcutaneous tissue exposed. Stage IV: muscle, tendon, bone or organs exposed. Bottom diagram showing unstageable pressure ulcer with tissue damage hidden from observer by eschar over entire wound. Deep tissue injury hidden from observer by intact skin appears as a bruise from above.

cotton-based fabrics due to a decrease in friction forces and subsequent damage to skin. ${ }^{26}$

Finally, adequate nutrition is very important in preventing pressure ulcer formation. Nutritional supplementation can benefit patients with limited oral intake and enteral or parenteral feeding can become necessary in patients who are not able to safely ingest enough oral nutrients. Protein intake is especially important to maintain a positive nitrogen balance, and vitamin/mineral supplements are recommended in patients lacking a balanced diet. ${ }^{27}$ Prealbumin is used as a laboratory test of short-term nutritional adequacy. Albumin is also useful, but its longer half-life means it is more reflective of nutrition over a long period of time.

\section{DIAGNOSIS AND ASSESSMENT}

Once a pressure ulcer is identified, staging and careful documentation of the size of the wound should be performed. Additional assessments of the ulcer include the location, surrounding skin condition, presence of tissue undermining and tunneling, and amount of exudate, odor, and tenderness. Pressure ulcers are classified into six categories (Fig. 2 and Table 2). ${ }^{7,28}$ Stage I describes intact skin with nonblanchable erythema. Stage II pressure ulcers have partial-thickness skin damage with possible blister formation, but no subcutaneous tissues visible. Stage III pressure ulcers have full-thickness skin loss with subcutaneous fat exposed but no muscles, bones, or tendons visible. Stage IV pressure ulcers have tissue loss with exposure of muscles, bones, tendons, or vital organs. A common hallmark of pressure ulcers is that the area of skin affected typically underestimates the amount of subcutaneous tissue involved. An unstageable pressure ulcer refers to a wound with an undetermined level of tissue injury because the entire base of the wound is covered by slough tissue and/ or eschar. A deep tissue injury is a term recently proposed by the National Pressure Ulcer Advisory Panel (NPUAP) to describe a pressure wound that has tissue injury hidden below intact skin. ${ }^{29}$ These wounds appear as deep bruises and have high potential for quick deterioration into a high-stage pressure ulcer.

The pressure ulcer scale for healing (PUSH tool) is a commonly used tool developed by the NPUAP,

Table 2. Table of different stages of pressure ulcers

Stage I

- Nonblanchable erythema

- Skin intact

Stage II

- Possible blister formation

- Partial-thickness skin damage

Stage III

- Subcutaneous fat exposed

- Full-thickness skin loss

Stage IV

- Exposed muscles, bones, tendons, or vital organs

- Skin, subcutaneous and possibly more tissue loss

Unstageable

- Entire wound base covered by slough and/or eschar

- Full-thickness skin loss

Deep tissue injury

- Unknown level of tissue injured below skin

- Skin intact 
Table 3. Pressure ulcer scale for healing (PUSH tool)

\begin{tabular}{ccll}
\hline Points & Area, $\mathrm{cm}^{2}$ (Length $\times$ Width) & \multicolumn{1}{c}{ Tissue Type } & Exudate Amount \\
\hline 0 & 0 & Closed & None \\
1 & $<0.3$ & Epithelial tissue & Light \\
2 & $0.3-0.6$ & Granulation tissue & Moderate \\
3 & $0.7-1.0$ & Slough & Heavy \\
4 & $1.1-2.0$ & Necrotic tissue & \\
5 & $2.1-3.0$ & & \\
6 & $3.1-4.0$ & & \\
7 & $4.1-8.0$ & & \\
8 & $8.1-12.0$ & & \\
9 & $12.1-24.0$ & & \\
10 & $>24.0$ & & \\
\hline
\end{tabular}

Copyright. NPUAP, 2003, reprinted with permission. Points are calculated per category and are added for a total score.

which grades pressure ulcers based on size of wound, wound bed tissue type, and exudate amount (Table 3). ${ }^{30}$ Another commonly used scale is the Bates-Jensen wound assessment tool which scores wounds based on size, depth, wound edges, tissue undermining, type and amount of necrotic tissue, type and amount of exudate, skin color, presence of edema, induration, granulation, and epithelialization. ${ }^{31}$ Other similar tools such as the pressure sore status tool and Sessing scale are also of use. ${ }^{32}$ It is more important to evaluate and monitor pressure ulcers in a close and consistent manner than the specific tool used to do so.

Further workup is sometimes warranted to define the extent of tissue involved. Because the area of skin breakdown is smaller than the total area affected, CT or MRI can be useful in defining the extent of the tissue involved and to determine whether osteomyelitis is present. ${ }^{33}$ Wound cultures are not routinely performed, but should be considered with lack of ulcer healing and persistent evidence of infection. If a wound culture is to be performed, tissue culture is more informative than simply performing a swab of the wound. ${ }^{5}$ Cultures showing more than $10^{5} \mathrm{CFU} / \mathrm{g}$ are indicative of active tissue infection. In the extremities, the adequacy of perfusion should be accessed via the ankle-brachial index and vascular studies. ${ }^{34}$

\section{TREATMENT}

The mainstays of pressure ulcer treatment include offloading the offending pressure source, adequate drainage of any areas of infection, debridement of devitalized tissue, and regular wound care to support the healing process.

\section{Pressure relief}

The first step in management is offloading pressure from the wound site. All of the measures described above for prevention of pressure ulcers are equally applicable to their treatment. For bedridden patients, this means strict adherence to repositioning the patient regularly. Any methods to pad the area of the pressure ulcer should be instituted if not already in use. Beyond the usual onlays and pads, some patients may require specialty beds to aid in decreasing pressure. These beds typically use air to continually shift pressure points through a variety of approaches. ${ }^{35}$ Even with these beds, patients still need to be repositioned regularly. For patients using prosthetics, they may need to go without them for a period of time to allow healing to occur. Any patient developing a pressure ulcer with a prosthetic should be refitted after they have healed to protect against future problems. Wheelchair-bound patients may need to have their mobility limited to allow healing. As with a prosthetic, the wheelchair should be reexamined for proper fit. ${ }^{36}$

\section{Infection control}

An important part of the initial evaluation of a pressure ulcer is to determine if there is evidence of inadequately treated infection. The pressure ulcer should be examined for the presence of surrounding erythema or fluctuance. The presence of crepitus is more ominous and should result in an expeditious assessment for the possibility of a necrotizing soft tissue infection. If there is a determination that there is inadequate source control, the patient should be taken to the operating room for appropriate abscess drainage and debridement.

Some surgeons elect to treat the wound initially with locally applied antiseptics, including povidone iodine, silver sulfadiazine, hydrogen peroxide, or Dakin's solution (sodium hypochlorite). The theory is that these topical agents serve to kill bacteria in the pressure ulcer to allow for better healing. If these solutions are used, they should only be used in the short term as they can also retard wound healing in the long term through their cytotoxic effects. ${ }^{37}$

Intravenous antibiotics should only be used in patients with significant cellulitis, or systemic signs and symptoms of infection, and should be stopped once those signs improve. A clean pressure ulcer, even with some necrotic debris, does not require intravenous antibiotics. Currently, most treatment protocols would recommend the use of intravenous antibiotics when there is evidence of osteomyelitis, but there is actually little evidence for its use. ${ }^{38,39}$ Generally, osteomyelitis requires debridement of the infected bone and coverage with a well-perfused flap to allow it to heal. ${ }^{40}$ Topical antibiotics have little role in the management of pressure ulcers. 
Table 4. Dressings available for pressure ulcer management with advantages, disadvantages, and ideal use

\begin{tabular}{llll}
\hline Type of Dressing & \multicolumn{1}{c}{ Advantages } & \multicolumn{1}{c}{ Disadvantages } & Ideal Wound \\
\hline Alginate dressings & Absorbent, infrequent changes & Expensive & Infected wounds \\
Foam dressings & Absorbent, provides padding & Expensive & $\begin{array}{c}\text { Infected wounds, fragile surrounding skin, Stage I } \\
\text { and for prevention }\end{array}$ \\
Gauze dressings & Inexpensive, microdebridement & Frequent changes & Large complex wounds with exudate or biofilm \\
Honey dressings & Mild antibiotic & Poor efficacy & Stage II with mild infection \\
Hydrocolloid dressings & Absorbent & Expensive & Wounds with minimal discharge, Stage II and III \\
Hydrogel dressings & Hydrating & Moves easily & Dry or dehydrated wounds, uninfected granulating wounds \\
Silver dressings & Antibiotic & Prevents epithelialization & Infected wounds, remove once infection is cleared \\
Transparent film dressing & Barrier from bodily fluids, & Not porous, can rip skin & Stage I, Stage II without exudate \\
& infrequent changes & on removal & \\
\hline
\end{tabular}

\section{Debridement}

Debridement of devitalized tissue and biofilm and abscess drainage are necessary in the treatment of pressure ulcers. In cases where there is a significant amount of necrotic tissue, performing the initial debridement in the operating room allows for a more definitive procedure. Subsequent debridements are then more easily managed at the bedside. There are instances where significant debridement is not needed or should not be done. If there is a dry eschar without purulence or fluctuance, and minimal erythema, the eschar can be left in place. If there is little subcutaneous tissue under the eschar, as in the case of the heel, debridement should be done with care. When performing surgical debridement, tissue should be resected until healthy bleeding tissue is encountered. After the initial presentation, repeated debridements are often necessary as the extent of necrosis can be difficult to assess. ${ }^{41}$

Other approaches to performing mechanical debridement include the use of acoustic energy in the form of ultrasound. Low-frequency ultrasound has been used to decrease bioburden of the wound and was shown to speed pressure ulcer healing. ${ }^{5} \mathrm{Hy}-$ drotherapy, including whirlpool, pulsed lavage, and vibration therapy are occasionally used for pressure ulcer debridement. There are additional products that perform an autolytic or enzymatic debridement over time when no urgent need for debridement exists. ${ }^{7}$ In cases where a patient cannot tolerate surgical debridement, there is an option of undergoing medical maggot debridement, in which maggots remove dead tissue, allowing the wound to heal. ${ }^{42}$ The goal of all these approaches is to create a bed of well-granulated tissue throughout the ulcer cavity. Small well-granulated ulcers can heal with re-epithelialization, while skin grafting or a surgical flap may be necessary in larger ulcers.

\section{Dressings and topical agents}

Dressings should be chosen depending on the wound being treated (Table 4 and Fig. 3 ). It should be noted that none of the dressings described below has been shown to have any superiority, and the choice of dressing should depend on the type of wound being treated. ${ }^{43}$ Things to be considered include size, depth, shape and location of the wound, presence and volume of exudate, presence of tunneling and tissue undermining, type of tissue in wound bed, and surrounding skin condition. Skin surrounding the ulcer should be protected from excessive moisture and friction to prevent breakdown.

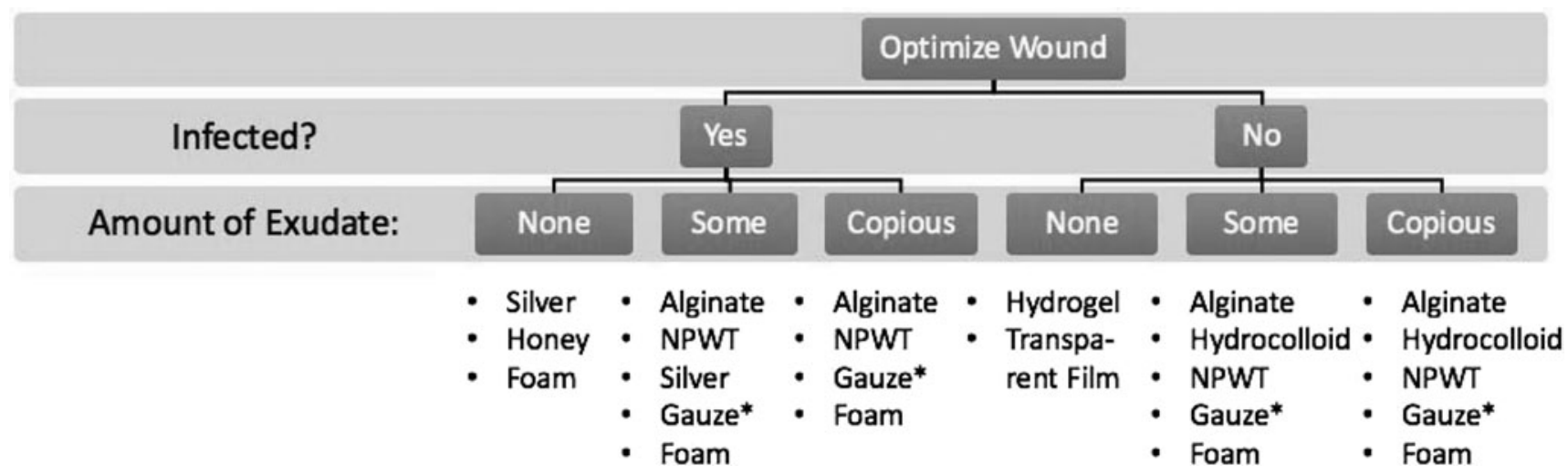

Figure 3. Algorithm for help in choosing an appropriate class of dressings for pressure ulcer management. *Gauze dressings can be used if limited options available require more frequent dressing changes. 
Dressings should be changed regularly and as soon as they become soiled with urine or feces to prevent wound contamination. Each dressing change should be accompanied by concurrent wound reassessment.

Gauze dressings. The traditional wet-to-dry method of gauze dressing now has more limited use in the treatment of pressure ulcers. While the materials are inexpensive, they do require frequent changes and the related nursing expense needs to be factored in when determining their true cost. ${ }^{44}$ When properly performed, they help maintain a moist wound environment, and the gauze also serves the role of performing a superficial debridement of biofilm and small amounts of necrotic tissue during dressing changes due to its adherent nature. ${ }^{45}$ The advent of advanced dressing materials makes gauze dressings a fallback when nothing else is readily available. ${ }^{46}$ Dry gauze dressings should not be used to treat pressure ulcers.

Alginate dressings. Alginate is a very absorbent material that is ideal for use in wounds with moderate to high discharge. They can absorb several times their weight in exudate and can conform well to irregular or tunneled wounds. Alginate dressings can be used in the setting of infected wounds and can be left in place longer than most dressings. ${ }^{47}$

Foam dressings. Foam dressings are made from polyurethane, a semipermeable material that can accommodate a medium to high amount of wound exudate and can be used in infected pressure ulcers. Foam dressings are often used for prevention of pressure ulcers because they provide some cushion. Silicone is often used in combination with foam dressings (such as Allevyn, Mepilex, and Optifoam) and is helpful in the setting of fragile tissue surrounding pressure ulcers. Dressings with silicone are less likely to cause trauma to skin on removal compared to other adherent dressings. ${ }^{48}$

Hydrocolloid dressings. Hydrocolloid dressings are made of a foam or film polyurethane material and contain a gelatin- or sodium carboxymethylcellulosebased gel material, which gives it the ability to absorb some fluids. They are well suited for wounds that have minimal to moderate drainage and are often used on Stage II and Stage III pressure ulcers. ${ }^{46}$

Hydrogel dressings. Hydrogel dressings are gel based and are 90\% water. These dressings are therefore ideally used in dry or dehydrated wounds and are often used over granulation tissue. In addition to being available in sheet form (where hydrogel is placed over a thin fiber mesh) and in the form of impregnated gauze, hydrogel also comes in its pure form in tubes and can be placed at the base of an uninfected granulating wound. This dressing should be covered by a sturdier dressing to prevent dislocation and dehydration of the hydrogel. ${ }^{49}$

Silver-containing dressings. Silver has bactericidal properties and dressings that are impregnated with silver are ideal for use in infected wounds. This dressing should be discontinued after clearance of infection as it can delay wound healing due to its toxicity to keratinocytes and fibroblasts. Silver is often incorporated into foam and alginate dressings. Silver alginate comes in rope and square forms, which are well suited for infected wounds with exudate, and gel form, which is better suited for drier wounds. ${ }^{50}$

Honey-containing dressings. There are anecdotal reports of the use of honey in the treatment of wounds since antiquity. In modern times, there is currently low evidence for the use of honey in the setting of pressure ulcers. ${ }^{28}$ Medical-grade honey has been shown to have mild antibiotic properties. Medical honey comes in stand-alone forms of gel or paste as well as impregnated into dressings where it is combined with alginate or hydrocolloid materials.

Transparent film dressings. Transparent film dressings are used primarily to protect Stage I or II ulcers where the skin remains intact. They provide a barrier to urine, stool, and other bodily fluids, which can macerate the skin. Because they are transparent and allow for observation of the wound, they can be applied and left in place for days. These dressings should not be applied in any ulcer where there is exudate as they are not porous. Care must be taken to remove these dressings as they can rip skin if removed forcefully. ${ }^{51}$

Negative pressure wound therapy. Negative pressure wound therapy (NPWT) consists of a foam dressing, which can be tailored to fit the patient's wound and is covered by a transparent film to enable creation of a vacuum in the wound when the foam is attached to a suction device via tubing. NPWT has been shown to speed wound healing in chronic wounds and the prevailing theory is that the vacuum causes the cells in the wound bed to sense a mechanical force. ${ }^{52}$ Mechanical forces stimulate the proliferation of fibroblasts leading to improved healing. The presence of the vacuum continuously eliminates exudate making it ideal in wounds where there is heavy exudate. Before application of the NPWT device, the wound must be 
adequately debrided. The foam dressing is easy to conform to wounds with unusual shapes, tunneling, and undermining. Because of the transparent film required for the vacuum to hold, NPWT is useful for preventing additional wound contamination. Randomized controlled trials showed no advantage of NPWT over other dressings. ${ }^{53}$ As with any dressing, its use is dictated by its properties. Wounds with a heavy exudate are readily managed with NPWT. It has been found to be helpful in wounds adjacent to fecal flow where its seal prevents wound contamination. Contraindications to NPWT use include uncorrected coagulopathy, exposed vital organs or large vessels. ${ }^{53}$ A nonadherent dressing can be placed below the foam to decrease pain on removal of foam dressing and during suctioning over the wound bed.

Other therapies. Biophysical treatments, including direct electric stimulation, pulsed electromagnetic field, and pulsed radio frequency energy, have been used to promote wound healing. Phototherapy treatment of pressure ulcers has been performed using laser, infrared, and ultraviolet waves. Studies have shown equivocal evidence concerning laser and infrared treatments, but ultraviolet $\mathrm{C}$ light therapy has been shown to decrease the bacterial burden and can be used following wound debridement in persistently infected wounds. ${ }^{54}$ Hyperbaric oxygen therapy and topical oxygen therapy have been used for pressure ulcer treatment with equivocal results. ${ }^{55}$

Biologic dressings describe a group of products derived from skin structures that have been purported to speed healing. They can be derived from nonhuman and human sources and are applied to noninfected, well-granulated wounds. The products range from decellularized human and porcine skin to dressings containing human fibroblasts and keratinocytes. Collagen is the main connective tissue fiber and application of collagen to uninfected granulating wound base can theoretically stimulate wound healing by providing a matrix network for cells to migrate into. These biologic dressings are typically used when an ulcer has fully granulated in, but still has a significant area of un-epithelialized wound. The dressings can be used in lieu of skin grafting to prevent having a second wound that needs attention. Growth factors have also been used by themselves to increase wound healing by stimulating angiogenesis and matrix deposition. ${ }^{56}$

\section{Patient optimization}

In addition to treatment of the pressure ulcer itself, it is important to treat the overall patient as well.
Physical measures to relieve pressure have already been described. Hyperglycemia will retard wound healing and diabetics should be aggressively treated to maintain glycemic control. The immune system has been shown to have a vital role in wound healing and immunosuppression will slow healing. Attention should be directed to any therapy that can cause immunosuppression, and these medications should be optimized to provide appropriate therapy without excessive immunosuppression. Proper wound healing requires adequate blood supply. For any pressure ulcer in the extremities, perfusion should be assessed and vascular surgery consulted if it is determined to be inadequate to support proper healing. ${ }^{5,7}$ Finally, we will stress once again the need to provide adequate nutrition. Periodic assessments of adequate nutrition should be performed by checking serum markers of nutrition such as albumin and prealbumin. Dietary intake should be adjusted to ensure the patient is in positive nitrogen balance. ${ }^{27}$

\section{Control of contamination}

By default, any open pressure ulcer is superficially contaminated with environmental flora. However, it is important to prevent added contamination if the wound is near the fecal stream as in ischial or sacral pressure ulcers. An added concern is that stool and urine can be irritating to the skin, causing further skin breakdown and extension of the ulcer. Because a large number of patients with pressure ulcers are incontinent of both bowel and bladder, it is important to consider how to deal with this. At the very least, these patients require frequent changes of their diapers to minimize skin contact with urine and stool. Consideration should be given to placement of a urinary catheter with the understanding that discomfort and complications, including urinary tract infection, are possible. Rectal tubes can be used but are rarely useful due to solid stools. ${ }^{57}$

Diversion of the fecal stream through surgical placement of a colostomy can be undertaken in those cases where it is felt to be necessary to allow proper healing. Any discussion of fecal diversion should include consideration of the likelihood that the colostomy will be permanent. Many of the patients who develop severe sacral and ischial pressure ulcers are incontinent or require a bowel program due to underlying conditions such as dementia or spinal cord injury. In these patients, having a permanent colostomy can actually be beneficial in their long-term care. ${ }^{58}$

\section{Surgery for reconstruction}

Although the majority of pressure ulcers will heal following debridement and conservative treatments 
outlined above, sometimes surgery will allow more rapid resolution of the ulcer. It should be noted that patients with poorly healing wounds should first be assessed for why the wound is not closing as those same factors could risk failure of surgical reconstruction. There are a variety of techniques available ranging from a simple skin graft to pedicled or, rarely, microvascular flaps for coverage. The appropriate candidate for surgical reconstruction has a wound that is without purulence, well-granulated and well-protected from soilage. The patient should be adequately nourished as assessed by nitrogen balance, albumin, and prealbumin, and without acute medical problems separate from the wound. Any intrinsic problems that can delay healing should be optimized, that is, well-controlled blood sugars in diabetics. In the appropriate patient, reconstruction will speed healing. Composite local tissue flaps are most frequently used to provide adequate tissue protection and perfusion. There are innumerable approaches to tissue coverage and the plan for each patient needs to be individualized. The flaps are planned so that suture lines remain away from pressure sites to ensure the best chance for healing. ${ }^{59}$ Microvascular tissue transfer is rarely used but indicated when local options for creating a flap are exhausted. ${ }^{60}$ Careful surgical planning is required to assure options for future reconstructions in cases of flap failure or pressure ulcer recurrence.

Among the indications for using surgical reconstruction are very large wounds, wounds with exposed organs and vessels, chronically nonhealing wounds, and wounds with osteomyelitis. Chronically infected bone will not allow appropriate healing of the overlying tissue. Prolonged antibiotics are often inadequate in curing the infection. A surgical approach to healing the ulcer requires debridement of the infected bone and placement of a well-vascularized flap to cover the area can allow the infection to be cured.

\section{SUMMARY}

Pressure ulcer prevention remains the most important step in the management of these wounds. However, despite best efforts, pressure ulcers may develop if enough risk factors are present. Treatment of pressure ulcers is necessary for patient comfort and to decrease risk of systemic infection.
The mainstays of treatment as outlined above include debridement of devitalized tissue, control of remaining infection with antibiotics, medical and nutritional patient optimization, appropriate dressing selection, and frequent monitoring of progression of wound evolution. If standard approaches are not adequate, additional therapies can be pursued, including biophysical modalities. Finally, in large ulcers, ulcers where wound healing is not adequately progressing, or ulcers where chronic osteomyelitis is present, reconstructive surgery can be considered.

\section{ACKNOWLEDGMENT AND FUNDING SOURCES}

The authors acknowledge generous support from the Hagey Laboratory for Pediatric Regenerative Medicine.

\section{AUTHOR DISCLOSURE AND GHOSTWRITING}

No competing financial interests exist. The content of this article was expressly written by the authors listed. No ghostwriters were used to write this article.

\section{ABOUT THE AUTHORS}

Tatiana V. Boyko, MD, is a general surgery resident at the University at Buffalo currently performing a postdoctoral research fellowship at Stanford University. Michael T. Longaker, MD, MBA, is the Deane P. and Louise Mitchell Professor of Surgery at Stanford University School of Medicine. He is the Director of the Hagey Laboratory for Pediatric Regenerative Medicine and the Co-Director of the Institute of Stem Cell Biology and Regenerative Medicine. George P. Yang, MD, PhD, is an Associate Professor of Surgery at Stanford University School of Medicine. 


\section{REFERENCES}

1. Odden MC, Coxson PG, Moran A, Lightwood JM, Goldman L, Bibbins-Domingo K. The impact of the aging population on coronary heart disease in the United States. Am J Med 2011;124:827-833.e5.

2. Murphy RA, Patel KV, Kritchevsky SB, et al Weight change, body composition, and risk of mobility disability and mortality in older adults: a population-based cohort study. J Am Geriatr Soc 2014;62:1476-1483.

3. Sen CK, Gordillo GM, Roy S, et al. Human skin wounds: a major and snowballing threat to public health and the economy. Wound Repair Regen 2009:17:763-771.

4. Coleman S, Nixon J, Keen J, et al. A new pressure ulcer conceptual framework. J Adv Nurs 2014;70:2222-2234.

5. Gould L, Abadir P, Brem H, et al. Chronic wound repair and healing in older adults: current status and future research. Wound Repair Regen 2015;23:1-13

6. Groeneveld A, Anderson M, Allen S, et al. The prevalence of pressure ulcers in a tertiary care pediatric and adult hospital. J Wound Ostomy Continence Nurs 2004;31:108-120; quiz 121-2.

7. Bluestein D, Javaheri A. Pressure ulcers: prevention, evaluation, and management. Am Fam Physician 2008;78:1186-1194.

8. Kosiak M. Etiology and pathology of ischemic ulcers. Arch Phys Med Rehabil 1959;40:62-69.

9. Kosiak M. Prevention and rehabilitation of pressure ulcers. Decubitus 1991;4:60-62, 64, 66 passim.

10. Walton-Geer PS. Prevention of pressure ulcers in the surgical patient. AORN J 2009;89:538-548; quiz 549-51.

11. Health Quality 0. Pressure ulcer prevention: an evidence-based analysis. Ont Health Technol Assess Ser 2009:9:1-104.

12. Kruger EA, Pires M, Ngann Y, Sterling M, Rubayi $S$. Comprehensive management of pressure ulcers in spinal cord injury: current concepts and future trends. J Spinal Cord Med 2013;36:572-585.

13. Verschueren JH, Post MW, de Groot $S$, van der Woude LH, van Asbeck FW, Rol M. Occurrence and predictors of pressure ulcers during primary in-patient spinal cord injury rehabilitation. Spinal Cord 2011:49:106-112.

14. Park-Lee E, Caffrey C. Pressure ulcers among nursing home residents: United States, 2004 NCHS Data Brief 2009:1-8.

15. Smith DM. Pressure ulcers in the nursing home. Ann Intern Med 1995;123:433-442.

16. Yip B, Stewart DA, Roberts MA. The prevalence of joint contractures in residents in NHS continuing care. Health Bull (Edinb) 1996;54:338-343.

17. Norton D. Calculating the risk: reflections on the Norton Scale. Decubitus 1989;2:24-31.
18. Bergstrom N, Braden BJ, Laguzza A, Holman V. The Braden Scale for predicting pressure sore risk. Nurs Res 1987;36:205-210.

19. European Pressure Ulcer Advisory Panel., National Pressure Ulcer Advisory Panel (U.S.). Prevention and Treatment of Pressure Ulcers: Clinical Practice Guideline: International Guideline. Washington, DC: National Pressure Ulcer Advisory Panel, 2009.

20. Park KH, Choi H. Prospective study on Incontinence-Associated Dermatitis and its Severity instrument for verifying its ability to predict the development of pressure ulcers in patients with fecal incontinence. Int Wound J 2016:13 Suppl 1:20-25.

21. Mclnnes E, Jammali-Blasi A, Bell-Syer SE, Dumville JC, Middleton V, Cullum N. Support surfaces for pressure ulcer prevention. Cochrane Database Syst Rev 2015;9:CD001735.

22. Reenalda J, Van Geffen P, Nederhand M, Jannink M, IJzerman M, Rietman H. Analysis of healthy sitting behavior: interface pressure distribution and subcutaneous tissue oxygenation. J Rehabil Res Dev 2009;46:577-586.

23. Giesbrecht EM, Ethans KD, Staley D. Measuring the effect of incremental angles of wheelchair tilt on interface pressure among individuals with spinal cord injury. Spinal Cord 2011;49:827-831.

24. Gunningberg L, Dahm MF, Ehrenberg A. Accuracy in the recording of pressure ulcers and prevention after implementing an electronic health record in hospital care. Qual Saf Health Care 2008;17:281285.

25. Thorpe E. Prophylactic use of dressings for pressure ulcer prevention in the critical care unit. $\mathrm{Br} \mathrm{J}$ Nurs 2016;25:S6-S12.

26. Twersky J, Montgomery T, Sloane R, et al. A randomized, controlled study to assess the effect of silk-like textiles and high-absorbency adult incontinence briefs on pressure ulcer prevention. Ostomy Wound Manage 2012;58:18-24.

27. Roberts $S$. Nutrition in pressure ulcer prevention: using a patient-centred approach. Old Nurse 2015;34:25.

28. Jull AB, Cullum N, Dumville JC, Westby MJ, Deshpande S, Walker N. Honey as a topical treatment for wounds. Cochrane Database Syst Rev 2015;3:CD005083

29. Ankrom MA, Bennett RG, Sprigle $S$, et al. Pressure-related deep tissue injury under intact skin and the current pressure ulcer staging systems. Adv Skin Wound Care 2005;18:35-42.

30. Choi EP, Chin WY, Wan EY, Lam CL. Evaluation of the internal and external responsiveness of the Pressure Ulcer Scale for Healing (PUSH) tool for assessing acute and chronic wounds. J Adv Nurs 2016;72:1134-1143.

31. Harris C, Bates-Jensen B, Parslow N, Raizman R, Singh $M$, Ketchen R. Bates-Jensen wound as- sessment tool: pictorial guide validation project. $J$ Wound Ostomy Continence Nurs 2010;37:253259 .

32. van Lis MS, van Asbeck FW, Post MW. Monitoring healing of pressure ulcers: a review of assessment instruments for use in the spinal cord unit. Spinal Cord 2010;48:92-99.

33. Bodavula P, Liang SY, Wu J, VanTassell P, Marschall J. Pressure ulcer-related pelvic osteomyelitis: a neglected disease? Open Forum Infect Dis 2015;2:ofv112.

34. Chiriano J, Bianchi C, Teruya TH, Mills B, Bishop V, Abou-Zamzam AM, Jr. Management of lower extremity wounds in patients with peripheral arterial disease: a stratified conservative approach Ann Vasc Surg 2010;24:1110-1116.

35. Clancy MJ. Pressure redistribution devices: what works, at what cost and what's next? J Tissue Viability 2013;22:57-62.

36. Gefen A. Tissue changes in patients following spinal cord injury and implications for wheelchair cushions and tissue loading: a literature review. Ostomy Wound Manage 2014;60:34-45.

37. Hidalgo E, Bartolome R, Dominguez C. Cytotoxicity mechanisms of sodium hypochlorite in cultured human dermal fibroblasts and its bactericidal effectiveness. Chem Biol Interact 2002;139:265-282.

38. Spellberg B, Lipsky BA. Systemic antibiotic therapy for chronic osteomyelitis in adults. Clin Infect Dis 2012;54:393-407.

39. Norman G, Dumville JC, Moore ZE, Tanner J Christie J, Goto S. Antibiotics and antiseptics for pressure ulcers. Cochrane Database Syst Rev 2016;4:CD011586.

40. Rennert R, Golinko M, Yan A, Flattau A, TomicCanic $M$, Brem $H$. Developing and evaluating outcomes of an evidence-based protocol for the treatment of osteomyelitis in Stage IV pressure ulcers: a literature and wound electronic medical record database review. Ostomy Wound Manage 2009;55:42-53

41. Schiffman J, Golinko MS, Yan A, Flattau A, TomicCanic $M$, Brem $H$. Operative debridement of pressure ulcers. World J Surg 2009;33:13961402

42. Felder JM, 3rd, Hechenbleikner E, Jordan M, Jeng $\mathrm{J}$. Increasing the options for management of large and complex chronic wounds with a scalable, closed-system dressing for maggot therapy. J Burn Care Res 2012;33:e169-e175.

43. Reddy M, Gill SS, Kalkar SR, Wu W, Anderson PJ, Rochon PA. Treatment of pressure ulcers: a systematic review. JAMA 2008;300:2647-2662.

44. San Miguel L, Torra i Bou JE, Verdu Soriano J. Economics of pressure-ulcer care: review of the literature on modern versus traditional dressings. J Wound Care 2007;16:5-9

45. Fleck CA. Why "wet to dry"? J Am Col Certif Wound Spec 2009;1:109-113. 
46. Zheng X, Li J. Comparison of the treatment of hydrocolloid and saline gauze for pressure ulcer: a meta-analysis of randomized controlled trials. Int J Clin Exp Med 2015;8:20869-20875.

47. Dumville JC, Keogh SJ, Liu Z, Stubbs N, Walker RM, Fortnam M. Alginate dressings for treating pressure ulcers. Cochrane Database Syst Rev 2015:5:CD011277.

48. Fletcher J. Understanding wound dressings: foam dressings. Nurs Times 2005;101:50-51.

49. Dumville JC, Stubbs N, Keogh SJ, Walker RM, Liu Z. Hydrogel dressings for treating pressure ulcers. Cochrane Database Syst Rev 2015;2: CD011226.

50. Silver dressings—-do they work? Drug Ther Bull 2010;48:38-42.

51. Dutra RA, Salome GM, Alves JR, et al. Using transparent polyurethane film and hydrocolloid dressings to prevent pressure ulcers. J Wound Care 2015;24:268, 270-261, 273-265.

52. Huang $C$, Leavitt $T$, Bayer LR, Orgill DP. Effect of negative pressure wound therapy on wound healing. Curr Probl Surg 2014;51:301-331.

53. Dumville JC, Webster J, Evans D, Land L. Negative pressure wound therapy for treating pressure ulcers. Cochrane Database Syst Rev 2015;5: CD011334.

54. Chen C, Hou WH, Chan ES, Yeh ML, Lo HL. Phototherapy for treating pressure ulcers. Cochrane Database Syst Rev 2014;7:CD009224.

55. Levine SM, Sinno S, Levine JP, Saadeh PB. Current thoughts for the prevention and treatment of pressure ulcers: using the evidence to determine fact or fiction. Ann Surg 2013;257:603-608.

56. Mudge EJ. Recent accomplishments in wound healing. Int Wound J 2015;12:4-9.
57. Ger R. Fecal diversion in management of large infected perianal lesions. Dis Colon Rectum 1996; 39:1327-1329.

58. Lee KF, Ennis WJ, Dunn GP. Surgical palliative care of advanced wounds. Am J Hosp Palliat Care 2007;24:154-160.

59. Levine SM, Sinno S, Levine JP, Saadeh PB. An evidence-based approach to the surgical management of pressure ulcers. Ann Plast Surg 2012;69:482-484.

60. Lemaire V, Boulanger K, Heymans 0 . Free flaps for pressure sore coverage. Ann Plast Surg 2008; 60:631-634.

\section{Abbreviations and Acronyms}

NPUAP $=$ National Pressure Ulcer Advisor Panel

NPWT $=$ negative pressure wound therapy 